\title{
ASSESSING VEGETATION STRUCTURAL CHANGES IN OASIS AGRO-ECOSYSTEMS USING SENTINEL-2 IMAGE TIME SERIES: CASE STUDY FOR DRÂA-TAFILALET REGION MOROCCO
}

\author{
L. Eddahby ${ }^{1 *}$, M.A. Popov ${ }^{2}$, S.A. Stankevich ${ }^{2}$, A.A. Kozlova ${ }^{2}$, M.O. Svideniuk ${ }^{2}$, D. Mezzane ${ }^{3}$, I. Lukyanchuk ${ }^{4}$, A. Larabi ${ }^{1}$ and H. \\ Ibouh $^{5}$ \\ ${ }^{1}$ Laboratory LIMEN “Water Resources \& Information Technology”, Mohammadia School of Engineers, BO 765 Agdal, Rabat, \\ Morocco (*DEVS/DSP in the ANDZOA, Rabat, Morocco) eddahbylhou1@gmail.com; \\ ${ }^{2}$ Scientific Centre for Aerospace Research of the Earth, Kiev, Ukraine - casre@,casre.kiev.ua; \\ ${ }^{3} \mathrm{LMCN}$, Faculty of Sciences and Techniques, Cadi Ayyad University, Marrakesh, Morocco; \\ ${ }^{4}$ LPMC, Picardie Jules Verne University, Amiens, France ; \\ ${ }^{5}$ LGSE, Faculty of Sciences and Techniques,Cadi Ayyad University, Marrakesh, Morocco - $\underline{\text { h.ibouh@uca.ma }}$
}

\section{Commission VI, WG VI/4}

KEY WORDS: LAI,Oasis, Vegetation Structure, Time Series Analysis, Change Detection, Remote Sensing, Sentinel-2, Tafilalet, Morocco

\begin{abstract}
:
Nowadays, Moroccan oasis agro-ecosystems are under intense effect of natural and anthropogenic factors. Therefore, this essay proposes to use Leaf Area Index (LAI) to assess the consequences of the oases long-term biodegradation. The index was used as a widely-applied parameter of vegetation structure and an important indicator of plant growth and health. Therefore, a new optical multispectral Sentinel-2 data were used to build a long term LAI time series for the area of the Erfoud and Rissani oases, Errachidia province in Drâa-Tafilalet region in Morocco. Nine images of LAI spatial distribution over the study area were obtained by means of SNAP Biophysical Processor over the period since July 2015 till May 2018. Time series analysis of the resultedmaps has revealed a stable trend towards the average LAI decreasing and vegetation structure simplification as a consequence.
\end{abstract}

\section{INTRODUCTION}

Oasis agro-ecosystems are of exceptional value for Moroccan natural heritage.In particular, covering about 15 percent of the country, these biodiversity hotspots provide crucial economic, ecological, social and cultural goods and services.However, numerous factors threat to the oases survival. Among them are the abandonment of traditional cultivation and farming systems that have nurtured the ecosystems for centuries. Secondly, increased pressure on land and water resources and growing urbanization support the negative effect. Moreover, desertification is an additional natural effect due to the Sahara intense expansion.(Bodart, et al;,2005)

Nowadays, the restoration of a sustainable oasis ecosystem is one of the main goals of the Moroccan Government and objective of international projects such as Revitalising Oasis Agro-ecosystems through a Sustainable, Integrated and Landscape Approach in the Draâ-Tafilalet Region (OASIL) (FAO and the GEF, 2017). Development of monitoring and evaluation indicators of oasis vegetation condition has a crucial significance for them.

Leaf area index (LAI) is considered an important crop parameter and an indicator of plant growth and health. It measures the amount of leaf material in an ecosystem and correlates directly with canopy foliage content and canopy structure (Borg, 2012). LAI is widely used for above-ground biomass assessment (Heiskanen, 2006), crop growth monitoring and yield estimation (Guindin-Garcia, 2012).

A broad variety of direct and indirect methods for remote estimation of the index has already proposed (Zheng, 2009 and Munier et al., 2018). Optical remote sensing methods build relationships exploiting in situ LAI measurements and/or as outputs of physical canopy radiative transfer models (Darvishzadeh, 2008). Novel European optical Sentinel-2 satellite provides perceptible advantages of spatial, spectral and temporal resolution for LAI estimation (Clevers, 2017). Moreover, European Space Agency trough Sentinels application platform (SNAP) proposes the processor that computing five main biophysical variables including LAI from Sentinel-2 reflectance (http://step.esa.int/main/toolboxes/sentinel-2toolbox/sentinel-2-toolbox-features/).

Given all the above, the objective of the present study was threefold: i) to explore the capability of LAI product obtained from Sentinel-2 to evaluate vegetation structure in oasis agroecosystems at the different stages of seasonal development; ii) to assess interannual changes of vegetation structure in oasis agro-ecosystems; iii) to identify a main trend of vegetation structural changes in studied ecosystems for the defined period.

\section{STUDY AREA AND METHODS}

\subsection{Study area}

The study area included the Erfoud and Rissani oases of Errachidia province in Drâa-Tafilalet region. These oases comprise numerous species of tree crops, cereals, vegetables, fodder, in addition to aromatic, dye and medicinal species (FAO and the GEF, 2017). The structure of the oases are formed mainly by date palm cultivation and arable parcels. In many oases a three-layer structure has been arranged. There upper layer is formed by date palms which protect from excessive insolation the fruit trees (figs, almonds, olives, pomegranates etc.) and the arable crops (barley, wheat, sorghum, alfalfa and various vegetables) of the two lower layers. Interacting in this 
way the layers contribute to the formation of a mild microclimate. A well-managed palm grove is vital for the maintaining the traditional oasis structure (Garbati Pegna, 2017).

Sand encroachment threating especially inland and coastal provinces: endangered palm groves are estimated at 30,000 ha (80,000 inhabitants) in the province of Ouarzazate (Bodart, et al;, 2005)and 250,000 ha (200,000 inhabitants)in the province of Errachidiaspecies (FAO and the GEF, 2017).

\subsection{Materials and Methods}

For the aim of the study nine Sentinel-2 L1C images for a period from 2015-2018 were acquired from COPERNICUS Sentinels scientific data hub (https://scihub.copernicus.eu).

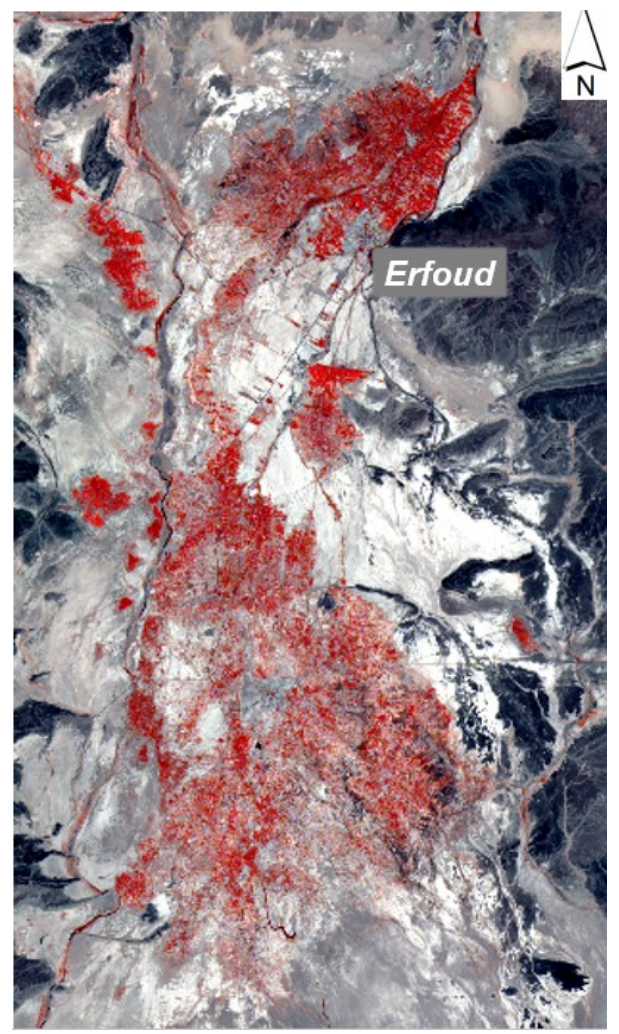

Figure 1. Sentinel-2 MSI $10 \mathrm{~m}$ resolution image of the area of interest. Data of image acquisition is July 16, 2015. Bands combination: $842 \mathrm{~nm}, 665 \mathrm{~nm}, 560 \mathrm{~nm}$

During each growing season, the dates of the images acquisition were defined according to annual distributions of average monthly temperature and precipitations. They are mid-term of May and November as start/end of the season with medium temperature and significant precipitations, and July as the hottest and driest period (https://weather-andclimate.com/average-monthly-Rainfall-Temperature-

$\underline{\text { Sunshine,erfoud-meknes-tafilalet-ma,Morocco ). }}$.

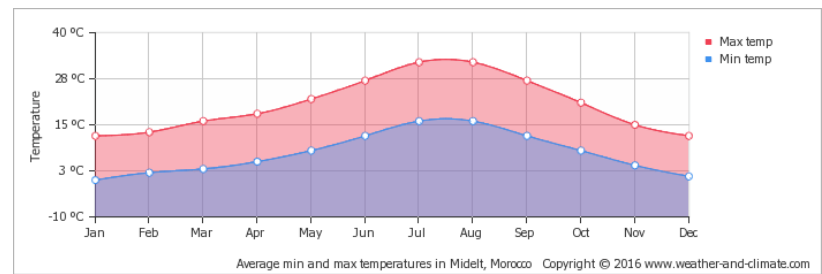

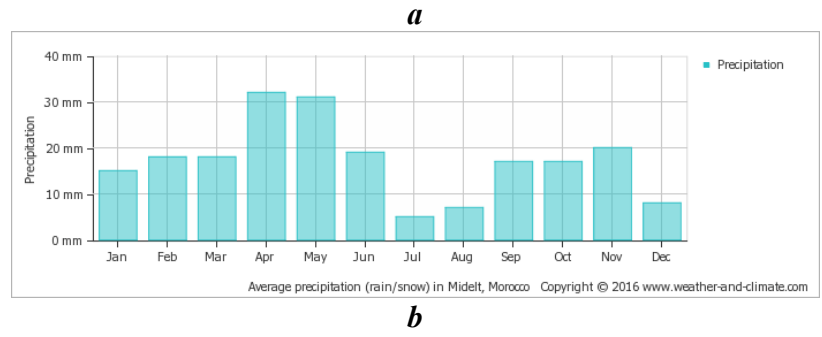

Figure 2. Climate indicators of the study area: a) average minimum and maximum temperature; b) average monthly precipitation over the year. Copyright (C) https://weather-andclimate.com

The input Sentinel-2 images were radiometrically and atmospherically corrected using SNAP Sen2Cor processor (http://step.esa.int/main/third-party-plugins-2/sen2cor/).

It performs the atmospheric-, terrain and cirrus correction of Top-Of- Atmosphere Level $1 \mathrm{C}$ input data. For each of preprocessed image LAlimage was derived by means of the Biophysical Processor. Its algorithm is based on specific radiative transfer models associated with strong assumptions, particularly regarding canopy architecture (turbid medium model) (Weiss, 2016).

Simultaneously, OSAVI (Optimized Soil-Adjusted Vegetation Index) was calculated and, applying the OSAVI threshold values, sparse vegetation of surrounding hills was masked out.This index was developed using the reflectance in the nearinfrared and red bands with an optimized soil adjustment coefficient (Rondeaux, 1996).OSAVI is robust to variability in soil brightness. It has enhanced sensitivity to vegetation cover greater than $50 \%$. This index is best used in areas with relatively sparse vegetation where soil is visible through the canopy and where NDVI saturates(Ren, 2014; Aralova, 2015; Santin-Janin, et al., 2018). 


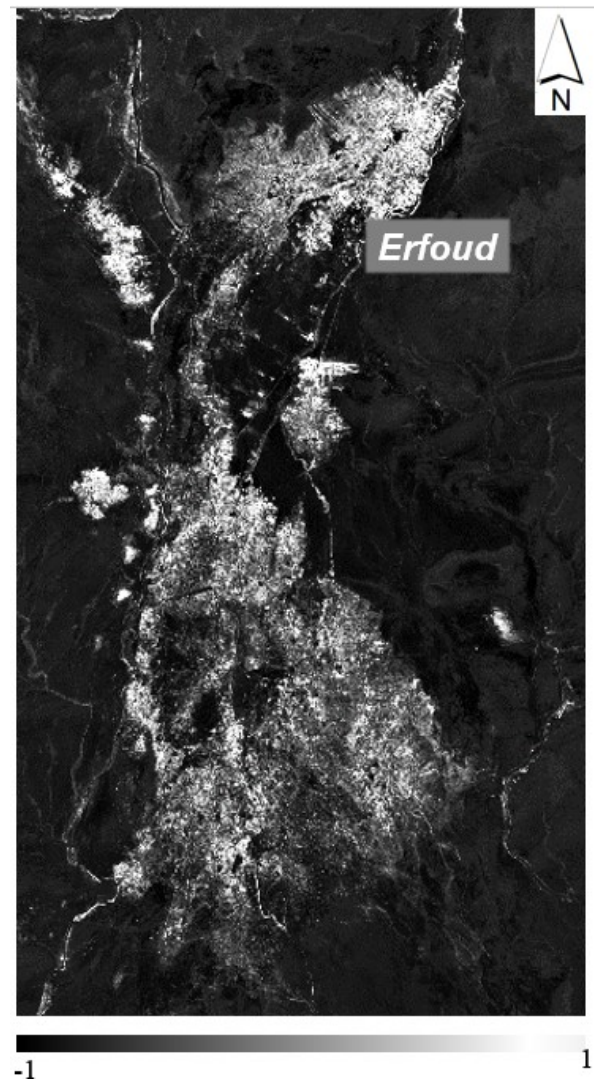

Figure 3. OSAVI calculated for the study area (16/07/2018). Vegetation is indicated by whit and light grey colors.

The obtained LAI distributions were under standard processing for time series analysis. The pixel-by-pixel simultaneous processing of all LAI maps resulted in spatial distributions of time series parameters, which describe the linear trend. These parameters are the all-time average and monthly rate.

The clearly expressed seasonal oscillations wereremoved from time series and the crisp linear trend was determined by the least squares method.

\section{RESULTS}

As a result, nine images of LAI spatial distribution over the study area were obtained. They are shown on Figure 4. Depending on vertical arrangement of leaf material that is corresponds to LAI values in the range from 0.2 to 5.0 , seven classes of vegetation structure in the oasis agro-ecosystems were determined(Table 1).
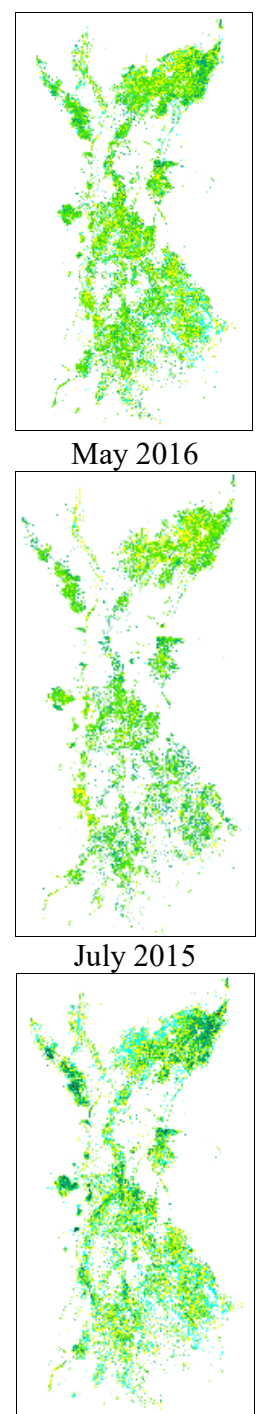

Nov 2015
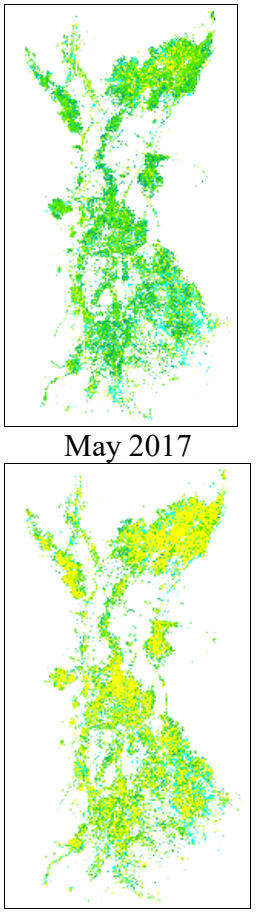

July 2016

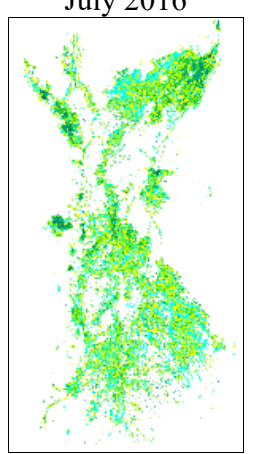

Nov 2016

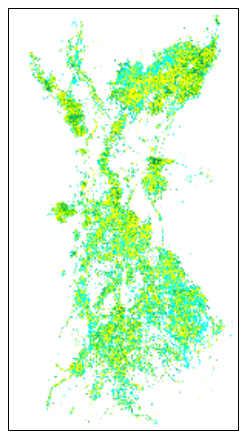

May 2018

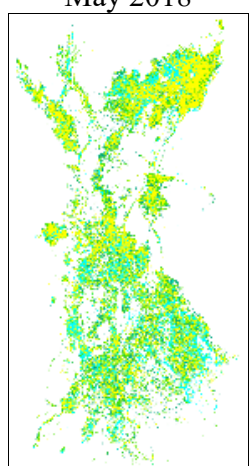

July 2017

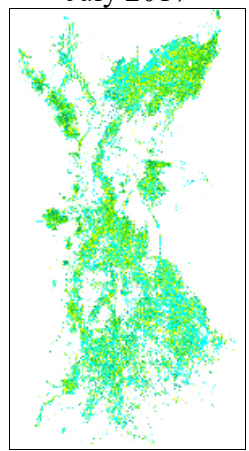

Nov 2017

0.2 to 0.4

0.4 to 0.6

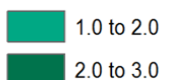

3.0 to 4.0

0.6 to 1.0

2.0 to 3.0

4.0 to 5.0

Figure 4. Spatial distributions of the within the study area over the period July 2015May 2018.

Obtained LAI maps shows that medium value classes have replaced by low value classeseach season since 2015 , while high value classes are critically small or disappeared. Thus, the maps describe the vegetation conditions dynamics and indicate vegetation structure simplification within the investigated oases. Areas of the distinguished seven classes over the period July 2015 toMay 2018 are presented in Table 1.

\begin{tabular}{|c|c|c|c|c|c|c|c|c|}
\hline \multirow[b]{2}{*}{ Date } & \multicolumn{8}{|c|}{ LAI Class } \\
\hline & $\begin{array}{l}0.2- \\
0.4\end{array}$ & $\begin{array}{l}0.4- \\
0.6\end{array}$ & $\begin{array}{l}0.6- \\
1.0 \\
\end{array}$ & $\begin{array}{l}1.0- \\
2.0\end{array}$ & $\begin{array}{l}2.0- \\
3.0 \\
\end{array}$ & $\begin{array}{l}3.0- \\
4.0 \\
\end{array}$ & $\begin{array}{l}4.0- \\
5.0\end{array}$ & Total \\
\hline $95 / 2018$ & 1440 & 5245 & 2085 & 503 & 31 & 2 & 0.03 & 9306 \\
\hline $11 / 2017$ & 6624 & 2741 & 2365 & 496 & 0 & 0 & 0 & 12226 \\
\hline $07 / 2017$ & 1556 & 5125 & 755 & 18 & 0 & 0 & 0 & 7454 \\
\hline p5/2017 & 119 & 1256 & 5218 & 2790 & 7 & 0.26 & 0.03 & 9390 \\
\hline $11 / 2016$ & 4116 & 4438 & 2519 & 1407 & 156 & 5 & 0.53 & 12642 \\
\hline $07 / 2016$ & 584 & 6569 & 1304 & 15 & 0 & 0 & 0 & 8472 \\
\hline $05 / 2016$ & 309 & 2421 & 6496 & 1789 & 42 & 2 & 0.06 & 11059 \\
\hline $11 / 2015$ & 3639 & 5032 & 3158 & 1957 & 339 & 21 & 1.22 & 14147 \\
\hline $07 / 2015$ & 118 & 1386 & 5691 & 2220 & 7 & 0.26 & 0.03 & 9422 \\
\hline
\end{tabular}

Table 1. Areas of LAI classes, ha 
A stable trend towards the average LAI decreasing was detected as a result of the time series analysis of the index multitemporal spatial distributions (Figure 5).

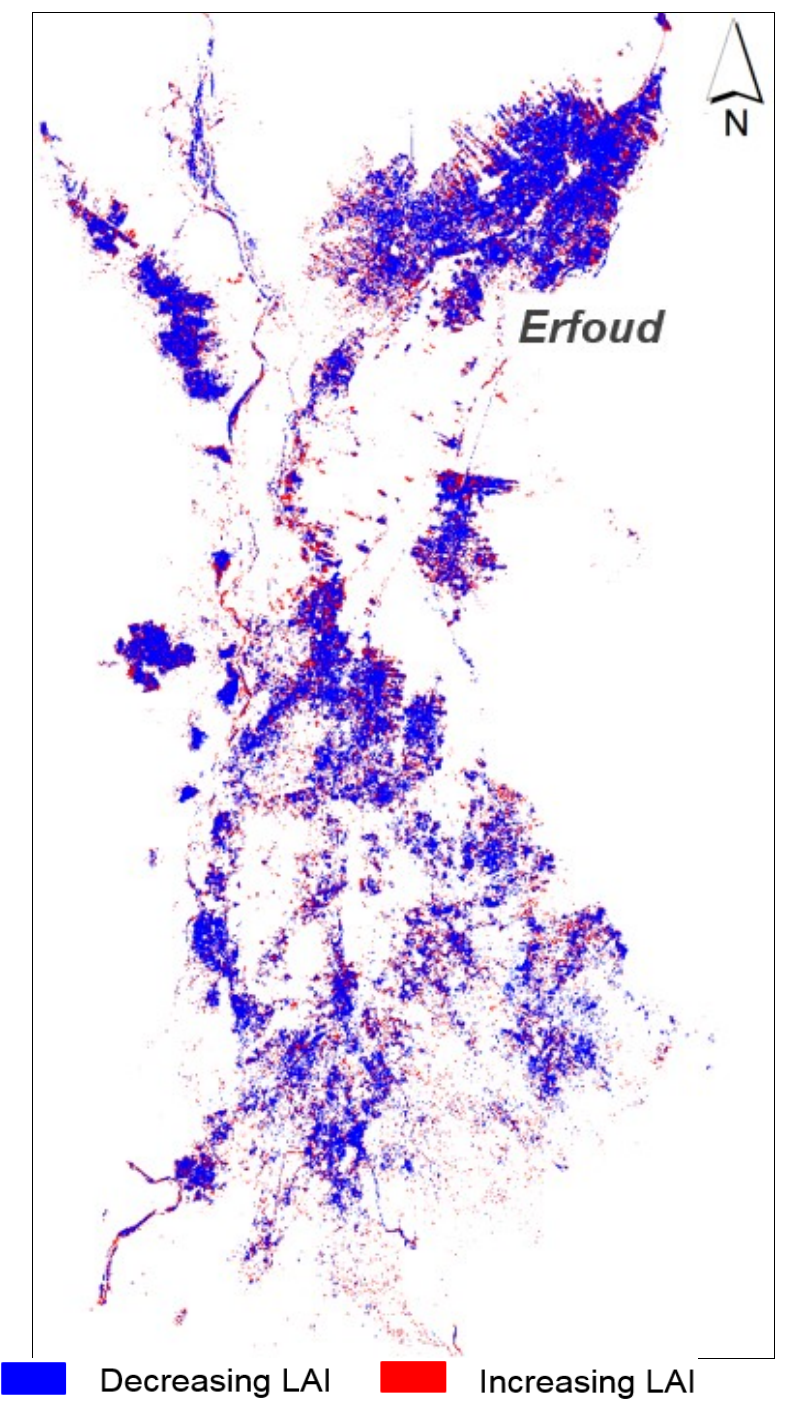

Figure 5. Total trend of LAI interannual changes within the study area over the period since July 2015 till May 2018.

Interannual development of LAI that embrace all seven classes together with a linear trend to LAI decreasing illustrate changes of vegetated areas andare showed in Figure 6.

Interannual changes of vegetated areas, ha

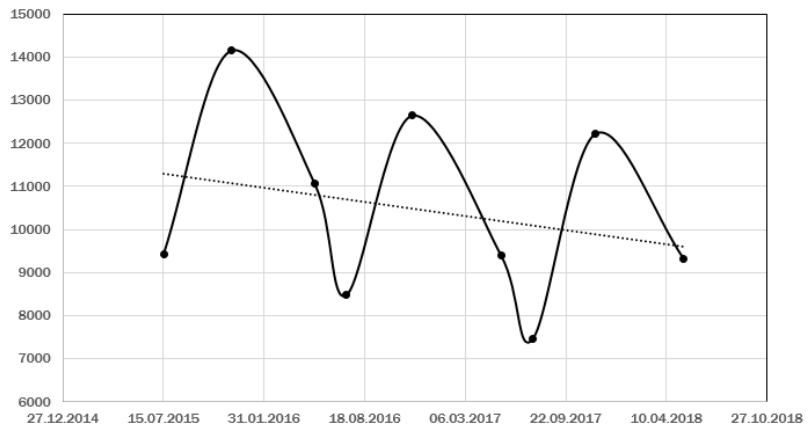

Figure 6. Interannual development of LAI and a resulting linear trend of changes in total vegetation amount.
From visual inspection of the results and comparison with highresolution imagery, the overall quality of class extraction seems to be satisfactory. Nevertheless, quantitative accuracy assessment is strongly required and should be performed in further research.

\section{CONCLUSIONS}

This research demonstrates that application of LAI product obtained from Sentinel-2 is very promising for spatio-temporal assessment of vegetation structure in oasis agro-ecosystems. The approach presented here will provide objective, reliable and operative information to oasis conservationist, managers and decision-makers on a character of structural changes in oasis vegetation. Further research should involve enhancement of LAI estimations by mean of in-situ measurements; refinement of vegetation structure classification taking into account crop types.

\section{REFERENCES}

Ahmadian, N., Borg, E., Roth, A., Zolitz, R., 2016. Estimating the Leaf Area Index of Agricultural Crops using multi-temporal dual-polarimetric, TerraSAR-X Data: A case study in NorthEastern Germany. Photogrammetry, Fernerkundung, Geoinformation, 2016. doi: 10.1127/pfg/2016/0307.

Aralova, D., Toderich, K., Osunmadewa, B. A., 2015. Spatial Distribution Patterns of Vegetation Cover in Deserts of Central Kyzylkum with application of Vegetation Indices (VIs). In: Journal of Arid Land 25-3:265 -268.

Bodart, C., Gassani, J., Salmon, M., \& Ozer, A 2006. Contribution of SAR Interferometry (from ERS-1/2) in the Study of the Aeolian Transport Processes: The Cases of Niger, Mauritania and Morocco. Fringe 2005 Workshop, 28 November- 2 December, 2005 in Frascati, Italy. Edited by H. Lacoste and L. Ouwehand. ESA SP-610. http://adsbit.harvard.edu//full/2006ESASP.610E..57B/0000057. $\underline{004 . h t m l}$

Clevers, J. G. P. W., Kooistra, L., Van Den Brande M. M. M., 2017. Using Sentinel-2 Data for Retrieving LAI and and Canopy Chlorophyll Content of a Potato Crop.In: Remote Sensing, 9(5), 405. doi:10.3390/rs9050405.

Darvishzadeh, R., Skidmore, A., Schlerf, M., Atzberger, C., 2008. Inversion of a radiative transfer model for estimating vegetation LAI and chlorophyll in a heterogeneous grassland. In: Remote Sensing of Environment. 112. 2592-2604. 10.1016/jrse.2007.12.003.

FAO and the GEF development team, 2017. Revitalizing oasis agro-ecosystems through a Sustainable, Integrated and Landscape Approach in the Draâ-Tafilalet Region (OASIL). Partnering for Sustainable Agriculture and the Environment. Available from:http://www.fao.org/gef/projects/detail/en/c $\underline{1056947 /}$

Garbati Pegna, F., P. Bartolini, L. El Rhaffari, S. Fahim, E. Bonaiuti, Q. B. Le, C. Zucca, 2017. Sustaining Moroccan oasis agricultural system through small mechanization inputs. / Migration. Peace and development. New challenges and new faces for Cooperation. V CONGRESS of the Italian University Network for Development Cooperation. / 14-15th of September. - Milan. $-10 \mathrm{p}$ 
Guindin-Garcia, N., Gitelson, A.A., Arkebauer, T.J., Shanahan, J., Weiss, A., 2012. Anevaluation of MODIS 8- and 16-day composite products for monitoring maizegreen leaf area index. Agric. For. Meteorol. 161, 15-25,

Heiskanen, J., 2006. Estimating aboveground tree biomass and leaf area index in a mountain birch forest using ASTER satellite data. In: International Journal of Remote Sensing, Vol.27(6)doi.org/10.1080/01431160500353858.

http://dx.doi.org/10.1016/j.agrformet.2012.03.012

$\underline{\text { http://step.esa.int/docs/extra/ATBD S2ToolBox L2B V1.1.pdf }}$

Munier S., Carrer D., Planque C., Camacho F., Albergel C. and Jean-Christophe Calvet, J.C., 2018. Satellite Leaf Area Index: Global Scale Analysis of the Tendencies Per Vegetation Type Over the Last 17. Years Remote Sens. 2018, 10, 424; doi $: 10.3390 /$ rs 10030424

Ren, H., Zhou, G., 2014. Determination of green aboveground biomass in desert steppe using litter-soil-adjusted vegetation index. In: European Journal of Remote Sensing, 47:1, 611-625, DOI: 10.5721/EuJRS20144734

Rondeaux, G., Steven, M., Baret, F., 1996. Optimization of soiladjusted vegetation indices. In: Remote Sensing of Environment. 55, pp 95-107

Santin-Janin, H.;. Garel M., Chapuis J. L., and Pontier D., (2018). Assessing the performance of NDVI as a proxy for plant biomass using non-linear models: a case study on the Kerguelen archipelago. Polar Biol (2009) 32:861-871. doi 10.1007/s00300-009-0586-5

Weiss, M., Baret, F., 2016. S2ToolBox Level 2 products: LAI, FAPAR, FCOVER. Version 1.1.

Zheng, G., Moskal M., 2009. Retrieving Leaf Area Index (LAI) using remote sensing: theories, methods and sensors. In:Sensors, 9, 2719-2745; doi:10.3390/s90402719. 